\title{
Information Visualization for Diabetes Management: A Literature Review
}

\author{
Yixuan Zhang* \\ zhang.yixua@northeastern.edu \\ Northeastern University
}

\author{
Andrea G. Parker \\ andrea@cc.gatech.edu \\ Georgia Institute of Technology
}

\author{
Cody Dunne \\ c.dunne@ northeastern.edu \\ Northeastern University
}

\begin{abstract}
Type 1 diabetes is a chronic illness that affects millions of people. People with type 1 diabetes regularly collect multidimensional data which they use to improve their well-being. Such data often includes blood glucose levels, insulin administration, diet, and physical activity. Monitoring and analysis tools for diabetes care often include information visualizations to help people make sense of this complex data. However, we have only an incomplete understanding of the visualization design approaches used or any justifications for the final design. To address this gap, we surveyed 21 diabetes data analysis tools which use visualization. From this, we derived a design space that consists of data, views, and strategies. We also provide design considerations for future researchers, tool designers, and developers.
\end{abstract}

\section{CCS CONCEPTS}

- Human-centered computing $\rightarrow$ Visualization; KEYWORDS

Personal informatics, Type 1 diabetes, visualization, sensemaking

\section{ACM Reference Format:}

Yixuan Zhang, Andrea G. Parker, and Cody Dunne. 2020. Information Visualization for Diabetes Management: A Literature Review. In 14th EAI International Conference on Pervasive Computing Technologies for Healthcare (PervasiveHealth '20), May 18-20, 2020, Atlanta, GA, USA. ACM, New York, NY, USA, 4 pages. https://doi.org/10.1145/3421937.3421957

\section{INTRODUCTION}

Type 1 diabetes is a chronic disease that affects millions of people. In diabetes management, patients are encouraged to regularly track diabetes-related data, such as blood glucose levels, insulin administration, diet, and physical activity. Patients are expected to regularly revisit and analyze their collected data so as to gain insights into necessary treatment adjustments. However, it is difficult to make sense of and analyze this complex, multidimensional, and often erroneous patient-logged data. Our discussion here of type 1 diabetes treatment is necessarily limited but we refer the interested reader to [29, 33].

Information visualization techniques have been commonly used to help make sense of diabetes data, including for identifying trends,

\footnotetext{
*Yixuan Zhang is now with Georgia Institute of Technology. Email: yixuan@ gatech.edu

Permission to make digital or hard copies of all or part of this work for personal or classroom use is granted without fee provided that copies are not made or distributed for profit or commercial advantage and that copies bear this notice and the full citation on the first page. Copyrights for components of this work owned by others than ACM must be honored. Abstracting with credit is permitted. To copy otherwise, or republish, to post on servers or to redistribute to lists, requires prior specific permission and/or a fee. Request permissions from permissions@acm.org.

PervasiveHealth '20, May 18-20, 2020, Atlanta, GA, USA

(C) 2020 Association for Computing Machinery.

ACM ISBN 978-1-4503-7532-0/20/05 . . \$15.00

https://doi.org/10.1145/3421937.3421957
}

patterns, and unusual occurrences [31]. Researchers have focused on many aspects of treatment such as the functional requirements in diabetes management [11] and the effect of app features on health outcomes [16]. However, there has been limited research examining the design of visualization techniques in currently available tools. In this paper, we contribute a literature review and analysis of existing diabetes tools. We examine how visualization techniques have been applied to support sensemaking using complex diabetes data. From this, we derive a design space consisting of data, views, and strategies supported by diabetes visualizations and provide design guidance for future researchers and practitioners.

\section{METHODOLOGY}

We reviewed the literature on type 1 diabetes tools with a focus on the design of any included visualizations. We first searched the ACM Digital Library, IEEE Xplore, and PubMed using the term "type 1 diabetes". For each of these papers, we expanded our search by examining the papers cited in each paper as well as any papers citing those already in our paper corpus. Additionally, we examined currently available apps supporting diabetes visualization by reviewing the Google Play Android store.

We included in our survey any papers/apps that: (1) were available in English, (2) included user interface examples as screenshots or linked videos and demos, and (3) support basic self-management tasks including monitoring blood glucose level and insulin dosage [10]. Additionally, mobile apps identified in the App store must: (4) be available for free download, (5) have been maintained and updated within the six months prior to our review (January-June 2019), and (6) function properly when used. This resulted in 9 academic papers $[4,7,14,15,20,24,25,30,33]$ and 12 mobile apps [1$3,5,8,9,18,22,23,26,27,32]$.

\section{FINDINGS}

Figure 1 shows the dominant features identified among the diabetes management visualizations we surveyed. We derived a design space consisting of Data, Views, and Strategies.

\subsection{Data \& Color Encodings}

We first summarize data being collected as this will be the first step for users to make sense of their diabetes data.

3.1.1 Data Sources. Current tools support showing multidimensional data from several sources. Data sources include continuous glucose monitors (CGM), self-monitoring of blood glucose (SMBG) data with finger stick testing, manual or automatic insulin administration logging, and other records about exercise, diet, body weight, multimedia (e.g., photos), and free-text notes that allow patients to 


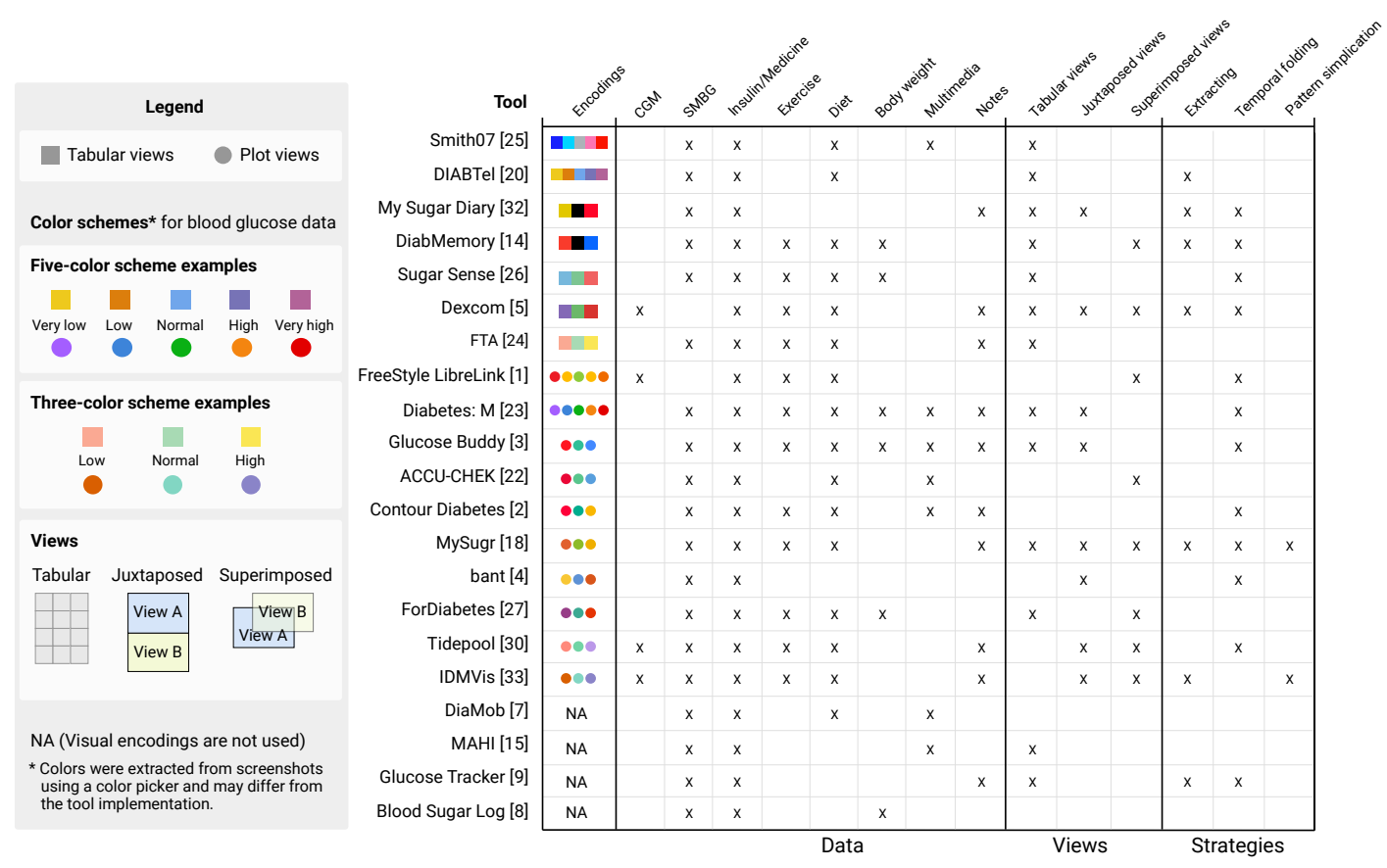

Figure 1: Mapping the surveyed tools into the design space of Data, Views, and Strategies

provide more contextual information. Among our surveyed tools, almost all of them (19) visualize SMBG data but only 4 display CGM data. It is notable that 2 of those visualize both CGM and SMBG data: Tidepool [30] and IDMVis [33]. All of them allow tracking insulin administration. Most tools (16) support tracking diet - e.g., carbohydrates, food ingredients, and nutritional information - and over half (12) support logging exercise. Only 6 tools allow users to input body weight. Only 7 tools support showing multimedia information (e.g., photos of meals) and only 10 support note-taking, both of which can aid recollection and reconstruction of event sequences. E.g., photos can help people to recall contextual information which further helps evaluate patients' diabetes status more accurately [7].

3.1.2 Color Encodings. Color is typically used to indicate various ranges of blood glucose levels from SMBG and CGM data. However, we found little consistency among current tools in the design of their color scales. Most (13) surveyed tools adopted a three-color scheme by dividing blood glucose levels into low, normal, and high ranges. E.g., a normal blood glucose range could be $70-180 \mathrm{mg} / \mathrm{dL}$ with anything under $70 \mathrm{mg} / \mathrm{dL}$ considered low and anything over 180 $\mathrm{mg} / \mathrm{dL}$ considered high [29]. 4 tools used a five-color scheme divide blood glucose levels into hypoglycemia (very low), low, normal, high, and hyperglycemia (very high). Colors were encoded either on plot views, e.g., on colored dots, (10 tools) or as part of table cells (7 tools). Despite the challenges in creating effective and accessible color scales for quantitative data [17], only one tool provided much rationale for the selected color scale: IDMVis [33]. The authors of IDMVis explained that the colors $(\bullet \bullet)$ were selected to help make extreme low and high blood glucose levels to pop out due to variation in lightness and to ensure accessibility by colorblind users.

\subsection{Views}

Chronic illness data reflects multiple facets of an individual's life. In diabetes care, the myriad data sources include both automatic collection by medical devices and manual text logs. However, users often track multi-sourced data using disparate platforms, such as using a CGM receiver or phone app (e.g., [5]) alongside a paper diabetes logbook. Prior work has explored approaches to supporting data integration and visualization of multiple dimensions of diabetes data simultaneously. Examples include incorporating blood glucose levels with digital photographs of meals [25], combining blood glucose levels and textual notes [30], and integrating and visualizing data from multiple medical devices and logbooks [33]. It can be challenging to visualize such complex and multidimensional diabetes data in a holistic fashion. Here, we analyze how current tools present their data and and categorize them as tabular, juxtaposed, and superimposed views.

3.2.1 Tabular Views. Tabular views display rows and columns of data [17]. People with diabetes are particularly familiar with tables as their logbooks commonly are ordered with time progressing throughout a day as you move to the right (e.g., breakfast, lunch, dinner, and bedtime columns) and across days as you move to the bottom. Among our surveyed tools, over half (13) use tabular views for blood glucose levels. The popularity of tables can be partially explained by a low cognitive cost of mental processing and the advantage of its familiarity [13]. Users are able to recognize outliers — e.g., unexpected low or high blood glucose values — by sorting by event type and color-coding blood glucose levels [13].

Any multimedia, text logs, or other contextual information is typically shown alongside the blood glucose readings in tabular views. Some tools provide interactive features to enable users to 
explore more contextual data, such as as hovering SMBG data points to see the attached photos or notes (e.g., [33]).

\subsubsection{Juxtaposed Views. Juxtaposed views place visualizations} side-by-side in one view [12] to allow exploration of relations among multiple data dimensions [19]. Many of our surveyed tools (8) used juxtaposition in their interface design. Example juxtaposed views include Tidepool [30] and Glucose Buddy [3], which place time series CGM visualizations in one view alongside another view of insulin administration, with both sharing a time axis. Juxtaposing different views allows interpreting blood glucose changes in context of other relevant data. Likewise, bant [4] and My Sugar Diary [32] showed trends for glucose readings of different events using juxtaposed views. Similarly, MySugr [18] and Dexcom [5] provided juxtaposed views to show blood glucose readings at various time frames similar to small multiples [28]. Each view in small multiples shares $\mathrm{x}, \mathrm{y}$, and size axes [21].

Other tools applied juxtaposition to help users explore the relationship between information shown in an overview and in a detailed view. For example, IDMVis [33] juxtaposes a 14-day overview with a more detailed 1-day view. Particular days could be interactively selected in the overview to update the detail view. Likewise, Diabetes:M [23] shows glucose history in an overview juxtaposed with an average glucose trend sorted by event types (e.g., meals).

3.2.3 Superimposed Views. Superimposed views overlay two visualizations on top of each other in a single view [12]. Using superimposition allows for easy comparison across different datasets as users do not need to jump between different parts of the screen. This can be particularly useful when visualizing chronic illness data that contains related data from multiple sources. IDMVis [33] is one example that superimposes CGM and SMBG readings to help identify issues of data quality such as incomplete or erroneous data. Additionaly, IDMVis superimposes meal logs and insulin administration amounts alongside the blood glucose data.

However, the use of superimposition in diabetes tools is quite varied. Tidepool [30] overlays insulin data and carbohydrates information in one view DiabMemory [14], FreeStyle LibreLink [1], ForDiabetes [27], and ACCU-CHEK [22] superimpose blood glucose readings with aggregate summary statistics (i.e., median, upper/lower quartile, and individual upper/lower alert indicators). Dexcom [5] superimposes blood glucose levels for an entire week using categorical to distinguish different days. MySugr [18] integrates insulin, blood glucose, and carbohydrate intake data into one view to allow reasoning about blood glucose changes. Superimposed views can also be used together with juxtaposed views. Tools like Tidepool [30], IDMVis [33], and MySugr [18] apply both juxtaposition and superimposition to visualize data from multiple sources.

\subsection{Strategies}

Guided by analytic focusing strategies proposed by Du et al. [6] for sensemaking using temporal event sequence data, we assessed how visualization has been used in the diabetes domain. We extracted features from our surveyed tools and mapped them to the relevant analytic strategies from Du et al.

3.3.1 Extraction Strategies. Extraction strategies focus the analysis on a subset of the data [6]. They are useful for identifying features linked to outcomes and investigating cause-and-effect relationships between events. Goal-driven record extraction, goal-driven event category extraction, and alignment are commonly-used strategies in data analysis [6]. Goal-driven record or event category extraction strategies focus on examining a subset of records or event types. Some of our surveyed tools applied these strategies. DIABTel [20] allows users to choose events of interest (e.g., blood glucose, diet, and insulin) to extract records that might be useful for sensemaking. Similarly, My Sugar Diary [32] allows for customizing events (e.g., bedtime and fasting) and updating the visualizations accordingly. MySugr [18] support extracting records and events, e.g., blood glucose records within a target range.

Alignment strategies aim to help understand precursor and aftereffect events to investigate cause-and-effect relationships [6] (e.g., "what happened to the blood glucose before and after lunch?"). For example, IDMVis [33] supports aligning records by a sentinel event of interest (e.g., lunch) to help identify precursor, intermediate, and aftereffect events. This alignment can help users explore trends and patterns as well as facilitate reasoning about treatment decisions.

3.3.2 Folding Strategies. Temporal folding strategies split long streams of data into smaller units such as individual days. This can help reduce pattern variety and expose cyclic or recurrent phenomena [6]. Over half (12) of the surveyed tools supported temporal folding but utilize different durations. Tidepool [30] allows users to switch between 1- and 7-day views. Alternately, bant [4] supports showing trends over 7-, 14-, 21-, and 90-day periods enabling different scopes of analysis. Likewise, MySugr [18] and FreeStyle LibreLink [1] allow users to switch between 7-, 14-, 30-, and 90-day periods Sugar Sense [26] provides visualizations for 7-, 30-, and 90-day periods. Contour Diabetes [2] shows daily, 7-, 30-, and all days. Glucose tracker [9] supports visualizing data for all days but separately the 1-day, 7-day, or monthly average. Diabetes:M [23] allows customization of the period to 1 day, week, month, or year. DiabMemory [14] allows users to freely customize the period by selecting start and end dates and times.

3.3.3 Pattern Simplification Strategies. Pattern simplification strategies focus on reducing variety by replacing complicated sequences with simpler ones. Two pattern simplification strategies have been applied among our surveyed tools, including grouping event categories and coalescing repeating point events into one. Grouping event categories requires aggregation of related events. For example, MySugr [18] groups event categories food types (e.g., dairy). These strategies allow exploration of patterns among filtered results, such as filtering by event types. Coalescing repeating point events typically converts multiple point events into one to simplify patterns. For example, IDMVis [33] and Tidepool [30] combine repeating CGM data to simplify the overall pattern and to optimize performance.

\section{DISCUSSION}

We propose directions and design considerations for future researchers and practitioners based on our findings.

Evaluate how well different views facilitate sensemaking of complex chronic illness data. Most of our surveyed tools used tabular views and some used juxtaposition and/or superimposition to aid understanding multidimensional data from multiple sources. Tabular 
views provides information in a more structured way to allow data comparison. Composite visualizations, alternately, allow users to compare data directly in the same visual space, but can cause visual clutter and decrease readability [12]. Therefore, evaluating how well different views facilitate sensemaking of diabetes data would help to advance our knowledge of designing diabetes visualizations.

Encourage consistency among color schemes and justify color choices. Current tools adopt color scales for blood glucose levels inconsistently and only one provided detailed justification for the color choices. We do not claim here that one color scheme would be best, but encourage designers, developers, and researchers to leverage best practices in color scale selection. We would hope that tool developers justify to themselves and to their users why and how a color scheme is selected to improve readability and accessibility.

Adopt analytic strategies to help sharpen the focus when analyzing multi-dimensional and complex chronic illness data. We adapted a set of analytic focusing strategies introduced by prior visualization researchers [6] to understand how current tools applied them to help users make sense of complex diabetes data. In particular, we mapped features of current tools into extraction, folding, and pattern simplification strategies. Temporal folding was more commonly used than extracting and pattern simplification. Temporal folding allows users to break long streams into shorter ones, such as splitting records into weekly and monthly views. Our surveyed tools provided a variety of folding options (e.g., 7-day, 14-day, 30day, 90-day, and all records). However, showing all records using superimposed views is likely to create high visual clutter, and thus make it challenging to analyze the the data. More work is needed to further explore the use of temporal folding techniques in composite visualizations, and how well various combinations of analytic strategies and views help people make sense of their data.

\section{CONCLUSION}

Our work examines how visualization techniques have been applied in current diabetes tools to help people make sense of complex and multidimensional diabetes data. We derived a design space consisting of data, views, and strategies in diabetes visualizations. The selection of the tools for this review may not fully cover the diabetes visualization design space thoroughly but we hope that our findings will assist future designers, developers, and researchers of diabetes visualization tools.

\section{REFERENCES}

[1] Abbott Laboratories, Inc. 2019. FreeStyle Libre.

[2] Ascensia, Inc. 2019. Contour Next. https://bit.ly/2kJEvrP

[3] Azumio, Inc. 2019. GlucoseBuddy. https://www.glucosebuddy.com/

[4] Joseph A Cafazzo, Mark Casselman, Nathaniel Hamming, Debra K Katzman, and Mark R Palmert. 2012. Design of an mHealth app for the self-management of adolescent type 1 diabetes: a pilot study. Journal of medical Internet research 14, 3 (2012), e70. https://doi.org/10.2196/jmir.2058

[5] Dexcom, Inc. 2019. Dexcom CLARITY Diabetes Management Software. https //www.dexcom.com/clarity

[6] Fan Du, Ben Shneiderman, Catherine Plaisant, Sana Malik, and Adam Perer 2017. Coping with Volume and Variety in Temporal Event Sequences: Strategies for Sharpening Analytic Focus. IEEE Trans. Vis. Comput. Graph. 23, 6 (2017), 1636-1649. https://doi.org/10.1109/TVCG.2016.2539960

[7] Dag Helge Frøisland and Eirik Årsand. 2015. Integrating visual dietary documentation in mobile-phone-based self-management application for adolescents with type 1 diabetes. Journal of diabetes science and technology 9, 3 (2015), 541-548.

[8] Futuretech Apps, Inc. 2019. Blood Sugar Log - Diabetes Tracker. https://play. google.com/store/apps/details?id=com.futuretech.diabetes.logs\&hl=en_US
[9] GlucoseTracker.net, Inc. 2019. GlucoseTracker. https://www.glucosetracker.net/

[10] S Goyal and JA Cafazzo. 2013. Mobile phone health apps for diabetes management: current evidence and future developments. QJM: An International Journal of Medicine 106, 12 (2013), 1067-1069. https://doi.org/10.1093/qjmed/hct203

[11] Can Hou, Ben Carter, Jonathan Hewitt, Trevor Francisa, and Sharon Mayor. 2016. Do mobile phone applications improve glycemic control (HbA1c) in the selfmanagement of diabetes? A systematic review, meta-analysis, and GRADE of 14 randomized trials. Diabetes Care 39, 11 (2016), 2089-2095. https://doi.org/10. 2337/dc16-0346

[12] Waqas Javed and Niklas Elmqvist. 2012. Exploring the design space of composite visualization. In 2012 IEEE Pacific Visualization Symposium. IEEE, 1-8. https: //doi.org/10.1109/PacificVis.2012.6183556

[13] Dmitri S Katz, Blaine A Price, Simon Holland, and Nicholas Sheep Dalton. 2018. Data, Data Everywhere, and Still Too Hard to Link: Insights from User Interactions with Diabetes Apps. In Proceedings of the 2018 CHI Conference on Human Factors in Computing Systems. ACM, 503. https://doi.org/10.1145/ 3173574.3174077

[14] Alexander Kollmann, Michaela Riedl, Peter Kastner, Guenter Schreier, and Bernhard Ludvik. 2007. Feasibility of a mobile phone-based data service for functional insulin treatment of type 1 diabetes mellitus patients. Journal of medical Internet research 9, 5 (2007), e36. https://doi.org/10.2196/jmir.9.5.e36

[15] Lena Mamykina, Elizabeth Mynatt, Patricia Davidson, and Daniel Greenblatt. 2008. MAHI: investigation of social scaffolding for reflective thinking in diabetes management. In Proceedings of the SIGCHI Conference on Human Factors in Computing Systems. ACM, 477-486. https://doi.org/10.1145/1357054.1357131

[16] Antonio Martinez-Millana, Elena Jarones, Carlos Fernandez-Llatas, Gunnar Hartvigsen, and Vicente Traver. 2018. App features for type 1 diabetes support and patient empowerment: systematic literature review and benchmark comparison. JMIR mHealth and uHealth 6, 11 (2018), e12237. https://doi.org/10.2196/12237

[17] Tamara Munzner. 2015. Visualization Analysis and Design. CRC Press.

[18] mySugr GmbH, Inc. 2019. MySugr. https://mysugr.com/en/

[19] Chris North and Ben Shneiderman. 1997. A taxonomy of multiple window coordination. Technical Report.

[20] Mercedes Rigla, M Elena Hernando, Enrique J Gómez, Eulalia Brugués, Gema García-Sáez, Verónica Torralba, Agustina Prados, Luisa Erdozain, Joana Vilaverde, and Alberto de Leiva. 2007. A telemedicine system that includes a personal assistant improves glycemic control in pump-treated patients with type 1 diabetes. Journal of diabetes science and technology 1, 4 (2007), 505-510. https://doi.org/ $10.1177 / 193229680700100408$

[21] George Robertson, Roland Fernandez, Danyel Fisher, Bongshin Lee, and John Stasko. 2008. Effectiveness of animation in trend visualization. IEEE transactions on visualization and computer graphics 14, 6 (2008), 1325-1332. https://doi.org/ 10.1109/TVCG.2008.125

[22] Roche Diabetes Care, Inc. 2019. Accu-Chek. https://www.accu-chek.com/

[23] Sirma Medical Systems, Inc. 2019. Diabetes:M. https://www.diabetes-m.com/

[24] Stein Olav Skrøvseth, Eirik Årsand, Fred Godtliebsen, and Gunnar Hartvigsen. 2012. Mobile phone-based pattern recognition and data analysis for patients with type 1 diabetes. Diabetes technology \& therapeutics 14, 12 (2012), 1098-1104. https://doi.org/10.1089/dia.2012.0160

[25] Brian K Smith, Jeana Frost, Meltem Albayrak, and Rajneesh Sudhakar. 2007. Integrating glucometers and digital photography as experience capture tools to enhance patient understanding and communication of diabetes self-management practices. Personal and Ubiquitous Computing 11, 4 (2007), 273-286. https: //doi.org/10.1007/s00779-006-0087-2

[26] StayWell, Inc. 2019. Sugar Sense - Diabetes App. https://play.google.com/store/ apps/details?id=org.medhelp.sugarsense

[27] Tessera Multimedia, Inc. 2019. forDiabetes: diabetes self-management app. https: //play.google.com/store/apps/details?id=gr.tessera.fordiabetesapp

[28] Edward R Tufte, Nora Hillman Goeler, and Richard Benson. 1990. Envisioning information. Vol. 126. Graphics press Cheshire, CT.

[29] Joseph I. Wolfsdorf. 2012. Intensive Diabetes Management (fifth edition ed.), American Diabetes Association.

[30] Jenise C Wong, Aaron B Neinstein, Howard Look, Brandon Arbiter, Nora Chokr, Cassie Ross, and Saleh Adi. 2017. Pilot Study of a Novel Application for Data Visualization in Type 1 Diabetes. Journal of diabetes science and technology 11, 4 (2017), 800-807. https://doi.org/10.1177/1932296817691305

[31] Ji Soo Yi, Youn-ah Kang, John T Stasko, and Julie A Jacko. 2008. Understanding and characterizing insights: how do people gain insights using information visualization?. In Proceedings of the 2008 Workshop on BEyond time and errors: novel evaLuation methods for Information Visualization. ACM, 4. https://doi.org/10.1145/1377966.1377971

[32] Ynapps, Inc. 2019. My Sugar Diary-Diabetes App. https://play.google.com/store/ apps/details?id=com.aiims.mysugardiary

[33] Yixuan Zhang, Kartik Chanana, and Cody Dunne. 2019. IDMVis: Temporal Event Sequence Visualization for Type 1 Diabetes Treatment Decision Support. IEEE transactions on visualization and computer graphics 25, 1 (2019), 512-522. https://doi.org/10.1109/TVCG.2018.2865076 